\title{
Areic Number
}

National Cancer Institute

\section{Source}

National Cancer Institute. Areic Number. NCI Thesaurus. Code C75752.

An expression of the number of entities per unit area. 